\title{
Viscoelasticity: an electrical point of view
}

\author{
Guido Ala*, Mario Di Paola ${ }^{\dagger}$, Elisa Francomano ${ }^{\ddagger}, \mathrm{Yan}_{\mathrm{Li}^{\S}}$ and Francesco P. Pinnola ${ }^{\dagger}$
}

\begin{abstract}
Time dependent hereditary properties of complex
materials are well described by power-laws with real order exponent. This experimental observation and analogous electrical experiments, yield a description of these properties by using fractional-order operators. In this paper, elasto-viscous and viscoelastic behaviors of fractional order hereditary materials are firstly described by using fractional mathematical operators, based on recent work of some of the authors. Then, electrical analogous models are introduced. Viscoelastic models have elastic and viscous components which can be obtained by combining springs and dashpots: these models can be equivalently viewed as electrical circuits, where the spring and dashpot are analogous to the capacitance and resistance, respectively. The proposed models are validated by using modal analysis. The use of electrical analogous in viscoelasticity can better reveal the real behavior of fractional hereditary materials.
\end{abstract}

*Università degli Studi di Palermo - DEIM, Viale delle Scienze Ed.9, I-90128 Palermo, Italy - guido.ala@unipa.it

†Università degli Studi di Palermo - DICAM, Viale delle Scienze Ed.8, I-90128 Palermo, Italy - mario.dipaola@unipa.it

‡Università degli Studi di Palermo - DICGIM, Viale delle Scienze Ed.6, I-90128 Palermo, Italy - elisa.francomano@unipa.it ${ }^{\S}$ School of Control Science and Engineering, Shandong University - Jinan, Shandong 250061, P. R. China - liyan.sdu@gmail.com

\section{MEChanicAl MODELS OF FHM}

Rubbers, polymers, bones, bitumen and many other materials, have a viscoelastic mechanical behavior [14]-[18]. The elastic behavior is typical of simple solid materials in which the strain history $\gamma(t)$ is linked by the stress history $\sigma(t)$ through a proportional relation as shown in Eq. (1):

$$
\sigma(t)=E \gamma(t)
$$

where $E$ is the Young modulus (Pascal). The equation Eq. (1) shows the so-called Hooke law, the mechanical model of elasticity is represented by a perfect spring with stiffness $E$ as shown in Fig. 1.

\section{INTRODUCTION}

The fractional calculus involves scientists belonging to various areas including viscoelasticity. [1]-[7]. In this context, mechanical models of fractional hereditary materials (FHM) have been introduced during the last century, in order to describe rheological stress-strain behavior in time domain. By classifying FHM as Elasto-Viscous (EV) and Visco-Elastic (VE), some of the authors have proposed both an exact and a discrete mechanical model of FHM [8], [9]. Moreover, the analogy between viscoelastic behavior and electrical elements constitutive equations is well-known so that, in spite of different physical meanings, the various used models, can also be applied to predict a circuit behavior [10]-[12]. In the paper, mechanical models of viscoelasticity behavior are firstly approached. Materials belonging to EV class are characterized by a dominant elastic behavior that leads to stress-strain relations involving fractional operators with index $\beta \in[0,1 / 2]$ ( $\beta=0$ is for a perfectly elastic behavior). VE materials show a dominant viscous behavior that leads to stress-strain relations with fractional operator order $\beta \in[1 / 2,1]$ ( $\beta=1$ is for of pure Newtonian fluid). So, EV material can be mechanically described, by an indefinite column of fluid resting on a bed of independent springs. VE material can be viewed by an indefinite shear type column resting on a bed of independent dashpots [8]. Discrete models of these continuous behaviors are depicted [9] and then, a general electrical analogous circuit is introduced in order to predict the behavior of FHM in an alternative way [13]. The proposed analogies are validated by using modal analysis.

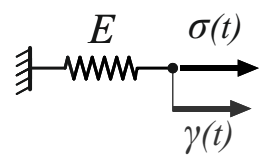

Fig. 1. Elastic model.

The viscous behavior is typical of a perfect fluid modeled by the Newtonian law, as shown in the following equation:

$$
\sigma(t)=\eta \frac{d}{d t} \gamma(t)=\eta \dot{\gamma}(t)
$$

where $\eta$ is the viscosity (Poise) of the fluid. In this case the stress history $\sigma(t)$ is related to the rate of deformation $\dot{\gamma}(t)$ and the model that describes this behavior is the dashpot shown in Fig. 2.

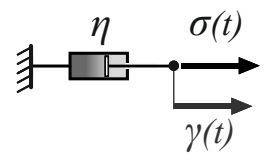

Fig. 2. Viscous model.

The models of Fig. 1 and Fig. 2 cannot be used to describe the viscoelastic behavior. So, various models have been introduced, in which the stress-strain relation is described with a differential relations [19], [20]. Moreover, from the relaxation function $G(t)$ representing the stress $\sigma(t)$ for an assigned strain history $\gamma(t)=H(t)$ (where $H(t)$ is the unit step function), and by using the Boltzmann superposition integral, the following stress-strain integral relation is obtained (valid for quiescent system at $t=0$ ):

$$
\sigma(t)=\int_{0}^{t} G(t-\bar{t}) d \gamma(\bar{t})=\int_{0}^{t} G(t-\bar{t}) \dot{\gamma}(\bar{t}) d \bar{t}
$$


Several experimental results show that $G(t)$ follows a powerlaw trend [21]-[24]:

$$
G(t)=\frac{C(\beta)}{\Gamma(1-\beta)} t^{-\beta}
$$

where $\Gamma(\cdot)$ is the Euler gamma function, $C(\beta)$ and $\beta$ are parameters that depend on the specific material. By using the relaxation function of Eq. (4) and applying the Boltzmann superposition integral of Eq. (3), another stress-strain relation can be obtained:

$$
\sigma(t)=C(\beta)\left({ }_{C} D_{0^{+}}^{\beta} \gamma\right)(t)
$$

where $\left({ }_{C} D_{0^{+}}^{\beta} \gamma\right)(t)$ is the Caputo's fractional derivative [25][28] of order $\beta$ of strain history $\gamma(t)$ with respect to time $t$, that is defined as:

$$
\left({ }_{C} D_{0^{+}}^{\beta} \gamma\right)(t)=\frac{1}{\Gamma(1-\beta)} \int_{0}^{t}(t-\bar{t})^{-\beta} \dot{\gamma}(\bar{t}) d \bar{t}
$$

The Eq. (5) shows the stress-strain relation of a new mathematical model of viscoelasticity that is known as spring-pot [29], [30]; the involved $\beta$ order of fractional operator is defined in the range $] 0,1[(\beta=0$ corresponds to pure elastic behavior of Eq. (1), while $\beta=1$ represents the pure viscous behavior of Eq. (2)).

Two different cases of viscoelasticity behavior can be distinguished: Elasto-Viscous, where $0<\beta<1 / 2$ and the elastic phase dominates; Visco-Elastic, where $1 / 2<\beta<1$ and viscous phase dominates [8].

For EV materials the exact mechanical model is a massless indefinite fluid column resting on a bed of independent springs as shown in Fig. 3, while for VE materials the exact mechanical model is a massless indefinite shear-type column resting on a bed of independent dashpot, Fig. 4.

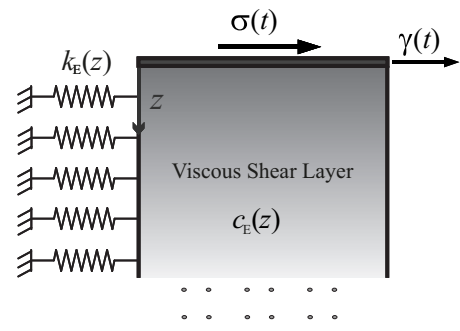

Fig. 3. Elasto-Viscous continuous fractional model.

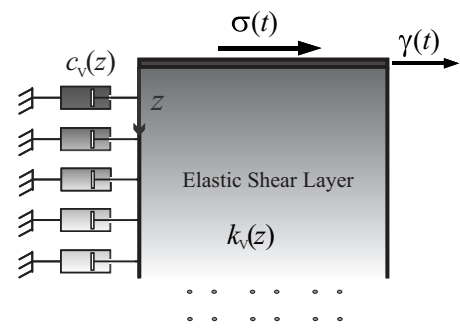

Fig. 4. Visco-Elastic continuous fractional model.

By introducing a $z$ coordinate, both for the EV and VE models, the fractional stress-strain relation of Eq. (5) is obtained at the top of the lamina if the following expressions are used as stiffness $k_{i}(z)$ and dashpot coefficients $c_{i}(z)(i=E$ for $\mathrm{EV}$ model and $i=V$ for $\mathrm{VE}$ model):

$$
\begin{aligned}
& (\mathrm{EV})\left\{\begin{array}{l}
k_{E}(z)=\frac{G_{0}}{\Gamma(1+\alpha)} z^{-\alpha}, \\
c_{E}(z)=\frac{\eta_{0}}{\Gamma(1-\alpha)} z^{-\alpha},
\end{array}\right. \\
& (\mathrm{VE})\left\{\begin{array}{l}
k_{V}(z)=\frac{G_{0}}{\Gamma(1-\alpha)} z^{-\alpha}, \\
c_{V}(z)=\frac{\eta_{0}}{\Gamma(1+\alpha)} z^{-\alpha},
\end{array}\right.
\end{aligned}
$$

By imposing the proper boundary conditions, the following expressions valid at the top of the lamina are obtained:

$$
\begin{array}{ll}
\text { (EV) } & \sigma(t)=C_{E}(\beta)\left({ }_{C} D_{0^{+}}^{\beta} \gamma\right)(t) \\
\text { (VE) } & \sigma(t)=C_{V}(\beta)\left({ }_{C} D_{0^{+}}^{\beta} \gamma\right)(t)
\end{array}
$$

where the coefficients $C_{E}(\beta)$ and $C_{V}(\beta)$ are defined as:

$$
\begin{aligned}
C_{E}(\beta) & =\frac{G_{0} \Gamma(\beta) 2^{2 \beta-1}}{\Gamma(2-2 \beta) \Gamma(1-\beta)}\left(\frac{\eta_{0} \Gamma(2-2 \beta)}{G_{0} \Gamma(2 \beta)}\right)^{\beta} \\
C_{V}(\beta) & =\frac{G_{0} \Gamma(1-\beta) 2^{1-2 \beta}}{\Gamma(2-2 \beta) \Gamma(\beta)}\left(\frac{\eta_{0} \Gamma(2-2 \beta)}{G_{0} \Gamma(2 \beta)}\right)^{\beta}
\end{aligned}
$$

Moreover, the mechanical models can be discretized and easily solved by a classical modal analysis [9]. The extension of fractional multiphase viscoelastic model can also be obtained [31], [32].

As it can be easily observed, the elastic law between stress and strain history modeled by a perfect spring, is analogous to Ohm law in which electric current $i(t)$ and voltage $v(t)$ are related by a linear relation; the Newtonian law is analogous to that of a purely capacitive element in which the electric current is related to the voltage temporal rate $\dot{v}(t)$. For an intermediate electrical behavior, between the purely capacitive and the purely resistive, a fractional current-voltage relation results. So a proper fractional electrical model of viscoelasticity can be straightforwardly obtained [13].

\section{FRACTIONAL ELECTRICAL CAPACITOR}

The well-known Curie's law [33] is an empiric relation between the current $i(t)$ and the applied voltage $v(t)=H(t)$. $V$ (being $H(t)$ the unit step function) of a real capacitor:

$$
i(t)=\frac{v(t) t^{-\beta}}{c(\beta) \Gamma(1-\beta)} \quad 0<\beta<1
$$

while $c(\beta)$ is a constant depending on the physical characteristics of the capacitor. $c(\beta)$ and $\beta$ can be obtained by experimental data. Eq. (10) can be viewed as the relaxation function. In fact, when $v(t)$ is applied to the capacitor, the corresponding $i(t)$ has a power law decay, then it relaxes. The current related to $V=1$ is denoted as $G_{E}(t)$ and can be called "Electrical-Relaxation Function" (ERF):

$$
\begin{aligned}
G_{E}(t) & =\frac{t^{-\beta}}{c(\beta) \Gamma(1-\beta)} ; & & t \geq 0,0<\beta<1 \\
G_{E}(t) & =0 ; & & t<0,0<\beta<1
\end{aligned}
$$


By applying the Boltzmann superposition principle, the current history in the capacitor due to a voltage $v(t)$ can be expressed as follows:

$$
i(t)=\int_{0}^{t} G_{E}(t-\bar{t}) \frac{d v(\bar{t})}{d \bar{t}} d \bar{t}
$$

The kernel of such a convolution integral is the ERF. Thus, the following relation is obtained:

$i(t)=\frac{1}{c(\beta) \Gamma(1-\beta)} \int_{0}^{t}(t-\bar{t})^{-\beta} \frac{d v(\bar{t})}{d \bar{t}} d \bar{t}=\frac{1}{c(\beta)}\left({ }^{c} D_{0^{+}}^{\beta} v\right)(t)$

When $\beta=1$ Eq. (13) restores an ideal capacitor of capacitance $C=1 / c(1)$; a pure resistor of resistance $R=c(0)$ results if $\beta=0$. If $0<\beta<1$ the fractional capacitor exhibits an intermediate behavior between an ideal resistor and an ideal capacitor. If a unit step current $i(t)=H(t) \cdot I$ with $I=1$ feeds the capacitor, the related voltage is denoted as $J_{E}(t)$ and can be called "Electrical-Creep Function" (ECF). By using again the Boltzmann superposition principle, the following relation holds:

$$
v(t)=\int_{0}^{t} J_{E}(t-\bar{t}) \frac{d i(\bar{t})}{d \bar{t}} d \bar{t}
$$

By applying the Laplace transform, the following relation between ERF and ECF results:

$$
\widehat{J}_{E}(s) \widehat{G}_{E}(s)=\frac{1}{s^{2}}
$$

$\widehat{J}_{E}(s)$ and $\widehat{G}_{E}(s)$ as the Laplace transforms of $J_{E}(t)$ and $G_{E}(t)$, respectively. By using equation Eq. (15), the ECF in time domain is obtained:

$$
\begin{aligned}
J_{E}(t) & =\frac{c(\beta) t^{\beta}}{\Gamma(1+\beta)}, & & t \geq 0 \\
J_{E}(t) & =0, & & t<0
\end{aligned}
$$

By inserting Eq. (16) in Eq. (14), it results:

$$
v(t)=\frac{c(\beta)}{\Gamma(1+\beta)} \int_{0}^{t}(t-\bar{t})^{\beta} \frac{d i(\bar{t})}{d \bar{t}} d \bar{t}
$$

After some trivial manipulations of the previous equation, it follows:

$$
v(t)=\frac{c(\beta)}{\Gamma(1+\beta)} \int_{0}^{t}(t-\bar{t})^{1-\beta} i(\bar{t}) d \bar{t}=c(\beta)\left(I_{0^{+}}^{\beta} i\right)(t)
$$

$\left(I_{0^{+}}^{\beta}\right)(t)$ is the Riemann-Liouville fractional integral. Power law of $J_{E}(t)$ or $G_{E}(t)$ shows that the fractional capacitor exhibits a long-tail memory [34], [35]. It can be called fractional hereditary capacitor. In the next section the electrical equivalent circuit of Eq. (13) is presented.

\section{ELECTRICAL EQUIVALENT OF FRACTIONAL CAPACITOR}

In this section a general electrical circuit model whose constitutive law is expressed by Eq. (13), is presented. By considering the elemental circuit of Fig. 5, the following diffusion equation holds:

$$
\frac{\partial}{\partial x}\left[\frac{1}{r(x)} \frac{\partial v(x, t)}{\partial x}\right]=c(x) \frac{\partial}{\partial t} v(x, t)
$$

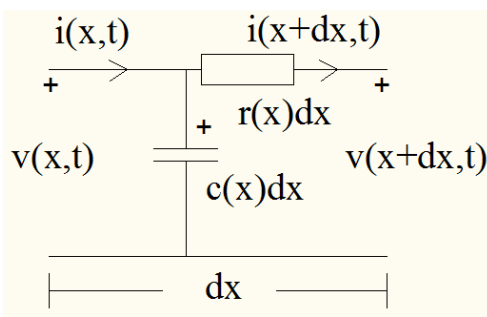

Fig. 5. General C-R electrical equivalent circuit.

For $\beta>1 / 2$ the coefficients of Eq. (19) are:

$$
\begin{aligned}
c(x) & =\frac{c}{\Gamma(1+\alpha)} x^{-\alpha} \\
r(x) & =r \Gamma(1-\alpha) x^{\alpha}
\end{aligned}
$$

where $\alpha=2 \beta-1$.

By inserting the coefficients of Eq. (20) in Eq. (19), it follows:

$$
\begin{aligned}
\frac{1}{r(x)} \frac{\partial^{2} v(x, t)}{\partial x^{2}}+\frac{1}{r^{\prime}(x)} \frac{\partial v(x, t)}{\partial x} & =c(x) \frac{\partial v(x, t)}{\partial t} \\
\frac{x^{-\alpha}}{r \Gamma(1-\alpha)} \frac{\partial^{2} v(x, t)}{\partial x^{2}}-\frac{\alpha x^{-1-\alpha}}{r \Gamma(1-\alpha)} \frac{\partial v(x, t)}{\partial x} & =\frac{c x^{-\alpha}}{\Gamma(1+\alpha)} \frac{\partial v(x, t)}{\partial t}
\end{aligned}
$$

so the following equation holds:

$$
\frac{\partial^{2} v(x, t)}{\partial x^{2}}-\frac{\alpha}{x} \frac{\partial v(x, t)}{\partial x}-\tau_{\beta} \frac{\partial v(x, t)}{\partial t}=0
$$

with $\tau_{\beta}=\operatorname{rc} \Gamma(2-2 \beta) / \Gamma(2 \beta)$.

By using Laplace transform, the following relation holds:

$$
\frac{d^{2}}{d x^{2}} V(x, s)-\frac{\alpha}{x} \frac{d}{d x} V(x, s)-\tau_{\beta} s V(x, s)=0
$$

where $V(x, s)=\mathcal{L}\{v(x, t), s\}$. Solution of Eq. (23) involves Bessel functions:

$$
V(x, s)=x^{\beta}\left[B_{1} \mathcal{I}_{\beta}\left(x \sqrt{s \tau_{\beta}}\right)+B_{2} \mathcal{K}_{\beta}\left(x \sqrt{s \tau_{\beta}}\right)\right]
$$

with $\mathcal{I}_{\beta}(\cdot)$ and $\mathcal{K}_{\beta}(\cdot)$ as the modified Bessel functions of first and second kind, respectively. By imposing the following boundary conditions:

$$
\begin{aligned}
& \lim _{x \rightarrow 0} V(x, s)=V(s) \Longrightarrow B_{2}=\frac{V(s)\left(\tau_{\beta} s\right)^{\beta / 2}}{\Gamma(\beta) 2^{\beta-1}} \\
& \lim _{x \rightarrow \infty} V(x, s)=0 \Longrightarrow B_{1}=0
\end{aligned}
$$

the following relation is obtained:

$$
\begin{aligned}
I(x, s) & =-\frac{1}{r(x)} \frac{d}{d x} V(x, s) \\
& =-\frac{V(s)}{r(x)} \frac{d}{d x}\left[\frac{\left(\tau_{\beta} s\right)^{\beta / 2}}{\Gamma(\beta) 2^{\beta-1}} x^{\beta} \mathcal{K}_{\beta}\left(z \sqrt{\tau_{\beta} s}\right)\right] \\
& =\frac{V(s)}{r(x)} \frac{\left(\tau_{\beta} s\right)^{\frac{1+\beta}{2}}}{\Gamma(\beta) 2^{\beta-1}} x^{\beta} \mathcal{K}_{\beta-1}\left(z \sqrt{\tau_{\beta} s}\right) \\
& =\frac{V(s)\left(\tau_{\beta} s\right)^{\frac{1+\beta}{2}}}{r \Gamma(2-2 \beta) \Gamma(\beta) 2^{\beta-1}} x^{1-\beta} \mathcal{K}_{\beta-1}\left(z \sqrt{\tau_{\beta} s}\right)
\end{aligned}
$$


in which $I(x, s)=\mathcal{L}\{i(x, t), s\}$. The fractional relation can be obtained by imposing the following:

$$
\begin{aligned}
\lim _{x \rightarrow 0} I(x, s)=I(0, s)=I(s) & =\frac{\Gamma(1-\beta) \tau_{\beta}^{\beta}}{r \Gamma(2-2 \beta) \Gamma(\beta) 2^{2 \beta-1}} V(s) s^{\beta} \\
& =\frac{1}{C_{C}(\beta)} V(s) s^{\beta}
\end{aligned}
$$

where:

$$
c_{c}(\beta)=\frac{r \Gamma(2-2 \beta) \Gamma(\beta)}{\Gamma(1-\beta) 2^{1-2 \beta}}\left(\frac{\Gamma(2 \beta)}{r c \Gamma(2-2 \beta)}\right)^{\beta}
$$

the subscript $c$ denotes that the system is more capacitive then resistive. By taking inverse Laplace transform of Eq. (27) it follows:

$$
i(t)=\frac{1}{c_{c}(\beta)}\left(D^{\beta} v\right)(t) .
$$

If $\beta=1 / 2 \Rightarrow \alpha=0 \Rightarrow c_{c}(1 / 2)=\sqrt{r / c}$ and Eq. (27) reduces to the following [13]:

$$
i(t)=\sqrt{c / r}\left(D^{1 / 2} v\right)(t)
$$

For the case $0<\beta<1 / 2$ the coefficients reported in Eq. (20) are modified as follows:

$$
\begin{aligned}
c(x) & =\frac{c}{\Gamma(1-\alpha)} x^{-\alpha} \\
r(x) & =r \Gamma(1+\alpha) x^{\alpha}
\end{aligned}
$$

with $\alpha=1-2 \beta$; in this case the coefficient of Eq. (28) becomes:

$$
c_{r}(\beta)=\frac{r \Gamma(2-2 \beta) \Gamma(1-\beta)}{\Gamma(\beta) 2^{2 \beta-1}}\left(\frac{\Gamma(2 \beta)}{r c \Gamma(2-2 \beta)}\right)^{\beta}
$$

In this case the system is more resistive than capacitive and the subscript $r$ denotes this behavior. By repeating the same procedure of the previous case, the following expression can be found:

$$
i(t)=\frac{1}{c_{r}(\beta)}\left(D^{\beta} v\right)(t) .
$$

Also in this case, if $\beta=1 / 2$ the Eq. (30) is verified.

\section{NUMERICAL VALIDATION}

A discretized version of the electrical circuit shown in Fig. 5 can be obtained by considering the C-R cell valid for a small but finite abscissa intervals $\Delta x$ [13]. By introducing a discretization of the $x$-axis as $x_{j}=j \Delta x$ into the governing Eq. (19) we get a finite difference equation as:

$$
\frac{\Delta}{\Delta x}\left[\frac{1}{r\left(x_{j}\right)} \frac{\Delta v\left(x_{j}, t\right)}{\Delta x}\right]=c\left(x_{j}\right) \dot{v}\left(x_{j}, t\right)
$$

so that, denoting $c_{j}=c\left(x_{j}\right) \Delta x$ and $r_{j}=r\left(x_{j}\right) \Delta x$, the continuos model is discretized into a lumped model with the following coefficients:

$$
r_{j}=r \Gamma(1-\alpha) \Delta x^{1+\alpha} j^{\alpha} ; \quad c_{j}=\frac{c \Delta x^{1-\alpha}}{\Gamma(1+\alpha)} j^{-\alpha}
$$

By supposing that the discretized circuit is driven by a current source $i_{s}(t)$, the following equations hold:

$$
\left\{\begin{array}{l}
c_{0} \dot{v}_{0}(t)-\frac{1}{r_{0}}\left(v_{1}(t)-v_{0}(t)\right)=i_{s}(t) \\
c_{1} \dot{v}_{1}(t)-\frac{1}{r_{2}}\left(v_{2}(t)-v_{1}(t)\right)+\frac{1}{r_{1}}\left(v_{1}(t)-v_{0}(t)\right)=0 \\
\vdots \quad \vdots \quad \vdots \\
c_{j} \dot{v}_{j}(t)-\frac{1}{r_{j+1}} \Delta v_{j+1}(t)+\frac{1}{r_{j}} \Delta v_{j}(t)=0
\end{array}\right.
$$

For simplicity's sake the case with $\beta=1 / 2$ is considered: so, the coefficients in the Eq. (36) become constants, $r_{j}=r \Delta x$ and $c_{j}=c \Delta x \forall j$, and the lumped circuit driven by current source becomes like the one shown in Fig. 6:

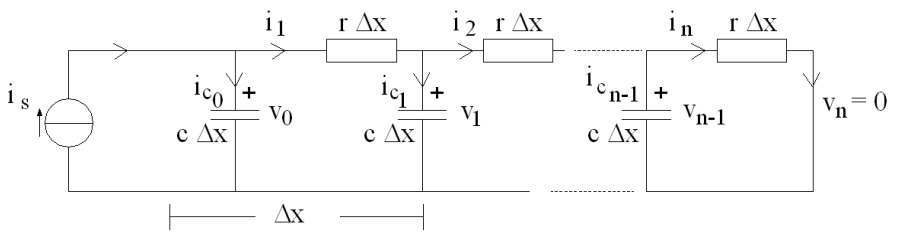

Fig. 6. Discretized model of electrical circuit shown in Fig. 5, driven by a current source

Let now introduce the node voltage $\boldsymbol{v}^{T}=\left[\begin{array}{lll}v_{0} & \cdots & v_{n-1}\end{array}\right]$ and the matrix of the corresponding node voltage temporal derivatives $\dot{\boldsymbol{v}}^{T}=\left[\begin{array}{lll}\dot{v}_{0} & \cdots & \dot{v}_{n-1}\end{array}\right]$, after some trivial manipulations the Eqs. (36) can be expressed in the following compact form [13]:

$$
\dot{\boldsymbol{v}}=\mathbf{A}_{i} \boldsymbol{v}+\frac{i_{s}}{c \Delta x} \mathbf{d}_{\mathbf{1}}
$$

where $\mathbf{d}_{1}^{T}=\left[\begin{array}{llll}1 & 0 & \cdots & 0\end{array}\right]$, and $\mathbf{A}_{i}$ is the following tridiagonal matrix

$$
\mathbf{A}_{i}=-\mu\left[\begin{array}{cccccc}
1 & -1 & 0 & 0 & \ldots & 0 \\
-1 & 2 & -1 & 0 & \ldots & 0 \\
0 & -1 & 2 & -1 & \ldots & 0 \\
\vdots & \ldots & \vdots & \ldots & \vdots & \ldots \\
0 & \cdots & 0 & -1 & 2 & -1 \\
0 & \cdots & 0 & 0 & -1 & 2
\end{array}\right]
$$

where $\mu=1 /\left[r c(\Delta x)^{2}\right]$. Since $\mathbf{A}_{i}$ is symmetric (and positive as definition), the matrix $\boldsymbol{\Phi}$ whose $j-t h$ column is the eigenvector $\phi_{j}$ leads to the following relations:

$$
\begin{aligned}
& \boldsymbol{\Phi}^{T}=\boldsymbol{\Phi} \\
& \boldsymbol{\Phi}^{T} \mathbf{A}_{i} \boldsymbol{\Phi}=-\mu \boldsymbol{\Lambda}
\end{aligned}
$$

where $\boldsymbol{\Lambda}$ is the diagonal matrix whose $j-t h$ element is the eigenvalue $\lambda_{j}$. By performing the following coordinates transform:

$$
\boldsymbol{y}=\boldsymbol{\Phi} \boldsymbol{v}
$$

in Eq. (37), and multiplying on the left by $\boldsymbol{\Phi}^{T}$, the following relation is obtained:

$$
\dot{\boldsymbol{y}}=-\mu \boldsymbol{\Lambda} \boldsymbol{y}+\frac{i_{s}}{c \Delta x} \boldsymbol{u}
$$

where $\boldsymbol{u}^{T}$ is the first row of the matrix $\boldsymbol{\Phi}$, namely:

$$
\boldsymbol{u}=\boldsymbol{\Phi}^{T} \mathbf{d}=\left[\begin{array}{llll}
\phi_{11} & \phi_{12} & \cdots & \phi_{1 n}
\end{array}\right]
$$


From equation (42) it results that in the modal space $y$ the differential equations are decoupled and are given in the form:

$$
\dot{y}_{j}(t)+\mu \lambda_{j} y_{j}(t)=\frac{\phi_{1 j}}{c \Delta x} i_{s}(t)
$$

and so:

$$
y_{j}(t)=y_{j}(0) e^{-\rho_{j} t}+\frac{\phi_{1 j}}{c \Delta x} \int_{0}^{t} e^{-\rho_{j}(t-\bar{t})} i_{s}(\bar{t}) d \bar{t}
$$

where $y_{j}(0)=\phi_{j}^{T} \boldsymbol{v}(0)$ and $\rho_{j}=\mu \lambda_{j}$. Once all the responses $y_{j}(t)$ are evaluated, the voltage vector $\boldsymbol{v}$ is readily formed as:

$$
\boldsymbol{v}=\boldsymbol{\Phi}^{T} \boldsymbol{y}
$$

the response in terms of voltage, which is related to fractional half derivative of the imposed current $i_{s}(t)$, is in the first term of the vector $\boldsymbol{v}$.

The eigenvalues $\lambda_{j}$ of matrix $\frac{-1}{\mu} \mathbf{A}_{i}$ and the corresponding eigenvectors $\phi_{j}$ normalized with the identity matrix, are:

$$
\begin{aligned}
& \lambda_{j}=2-2 \cos \left(\frac{2 j-1}{2 n+1} \pi\right) ; \quad j=1,2, \ldots, n \\
& \phi_{k, j}=\sqrt{\frac{4}{2 n+1} \cos \left[\frac{(2 j-1)(2 k-1) \pi}{2(2 n+1)}\right] ; j, k}=1,2, \ldots, n
\end{aligned}
$$

where $\phi_{k, j}$ is the $k-t h$ component of the vector $\phi_{j}$.

As an example, let us suppose that the imposed current is expressed by $i_{s}(t)=H(t)$, by using the introduced method to modeling the fractional capacitors and the aforementioned modal analysis the response in terms of voltage is found. The Fig.7 shown the voltage response and the comparison between the approximate solution, that is obtained by the discrete model of capacitor, and the well known exact solution of this problem in terms of ECF.

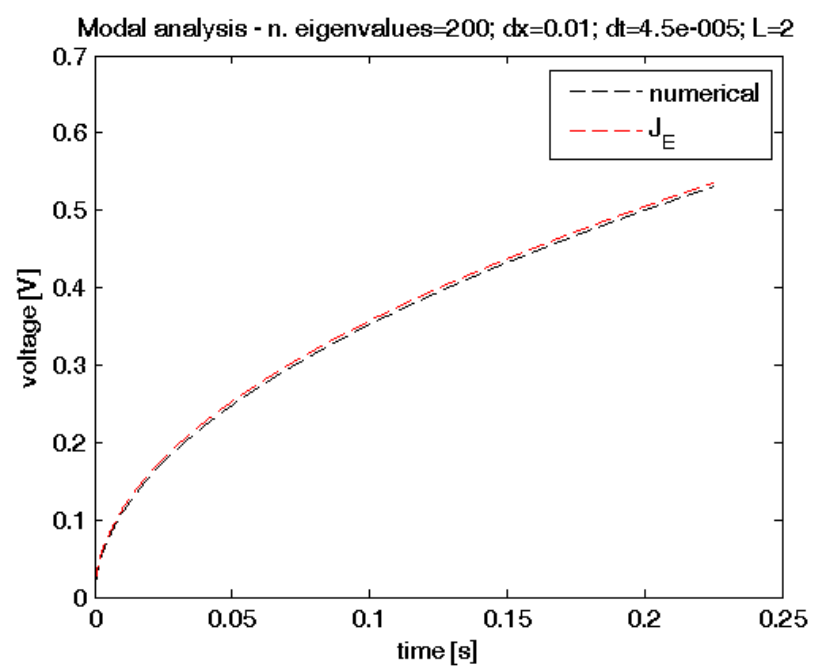

Fig. 7. Comparison between modal analysis results and ECF profile: nodal voltage $v_{1}$

\section{CONCLUSION}

Mechanical models of materials viscoelasticity behaviour have been approached by using fractional calculus. Then, a general electrical analogous model of fractional hereditary materials has been introduced. In fact, viscoelastic models have elastic and viscous components which can be obtained by combining springs and dashpots: these models can be equivalently viewed as electrical circuits, where the spring and dashpot are analogous to the capacitance and resistance, respectively. The proposed model has been validated by using modal analysis. The use of electrical analogous in viscoelasticity can help to better investigate the real behavior of fractional hereditary materials.

\section{ACKNOWLEDGMENT}

Support by Universita' degli Studi di Palermo is gratefully acknowledged.

\section{REFERENCES}

[1] Yang Z., Cao J., Initial value problems for arbitrary order fractional differential equations with delay, Communications in Nonlinear Science and Numerical Simulation, Volume 18, n.11, pp. 2993 - 3005, 2013.

[2] Liu Y., Application of AveryPeterson fixed point theorem to nonlinear boundary value problem of fractional differential equation with the Caputos derivative, Communications in Nonlinear Science and Numerical Simulation, Volume 17, n.12, pp. 4576 - 4584, 2012.

[3] Machado J. T., Kiryakova V., Mainardi F., Recent history of fractional calculus, Communications in Nonlinear Science and Numerical Simulation, 16 (3), pp. 4756 - 4767, 2011.

[4] Bouafoura M. K., Braiek N. B., Controller design for integer and fractional plants using piecewise orthogonal functions, Communications in Nonlinear Science and Numerical Simulation, Volume 15, n.5, pp. 1267 - 1278, 2010

[5] Petras I., Sierociuk D., Podlubny, I., Identification of parameters of a halforder system, IEEE Transactions on Signal Processing, 60 (10), pp. 55615566, 2012.

[6] Podlubny, I., Petras, I., Vinagre, B.M., O'Leary P., Dorcak L., Analogue Realizations of Fractionalorder Controllers, Nonlinear Dynamics, 29 (14), pp. 281296, 2002.

[7] Caponetto R., Dongola G., Fortuna L., Petr I., Fractional Order Systems: Modeling and Control Applications, World Scientific Series on Nonlinear Science Series A: Volume 72, 2010.

[8] Di Paola M., Zingales M., Exact mechanical models of fractional hereditary materials, Journal of Rheology, Volume 56, Issue 5, pp. 9831004, 2012.

[9] Di Paola M, Pinnola F. P., Zingales M., A discrete mechanical model of fractional hereditary materials, Meccanica: An International Journal of Theoretical and Applied Mechanics, Volume 48 (7), pp. 1573-1586, 2013

[10] Gross B., Electrical analogs for viscoelastic systems, Journal of Polymer, Science, 20 (95), pp. 371-380, 1956.

[11] Carlson G., Halijak C., Approximation of fractional capacitors $(1 / s)^{(1 / n)}$ by a regular newton process, IEEE Transactions on Circuit Theory, 11 (2), pp. 210-213, 1964.

[12] Westerlund S., Ekstam L., Capacitor theory, IEEE Transactions on Dielectrics and Electrical Insulation, 1 (5), pp. 826-839, October 1994.

[13] Ala G., Di Paola M., Francomano E., Li Y., Pinnola F. P., Electrical analogous in viscoelasticity, Communications in Nonlinear Science and Numerical Simulation, vol. 19 (7), pp. 2513-2527, 2014.

[14] Jerri A., Introduction to integral equations with applications, Wiley, New York, 1999.

[15] Bates J.H.T., Lung Mechanics. An Inverse Modeling Approach, Cambridge University Press, 2009.

[16] Ionescu C.M., De Keyser R., Relations Between Fractional-Order Model Parameters and Lung Pathology in Chronic Obstructive Pulmonary Disease, IEEE Transactions on Biomedical Engineering, Volume 56, n. 4, pp. 978-987, 2009. 
[17] Craiem D. O., Armentano R. L., Arterial viscoelasticity: a fractional derivative mode, Proceedings of 28-th IEEE EMBS Annual International Conference, New York City, USA, Aug 30-Sept 3, 2009.

[18] Valdez-Jasso D., Haider M.A., Banks H. T., Santana D.B., German Y.Z., Armentano R.L., Olufsen M.S., Analysis of Viscoelastic Wall Properties in Ovine Arteries, IEEE Transactions on Biomedical Engineering, Volume 56, n. 2, pp. 210-219, 2009.

[19] Flügge W, Viscoelasticity, Blaisdell Publishing Company, Waltham, 1967.

[20] Christensen R. M., Theory of Viscoelasticity, Academic Press, New York, 1982.

[21] Nutting P. G., A new general law of deformation, Journal of the Franklin Institute, Volume 191, pp. 679-685, May 1921.

[22] Gemant A., A method of analyzing experimental results obtained from elasto-viscous bodies, Physics, Volume 7, pp. 311-317, August 1936.

[23] Deseri, L, Di Paola, M., Zingales, M., Pollaci, P., Power-law hereditariness of hierarchical fractal bones, International Journal for Numerical Method Biomedical Engineering, Invited paper, article in press, DOI: $10.1002 / \mathrm{cnm}$.

[24] Di Paola, M., Fiore, V., Pinnola, F. P., Valenza, A., On the influence of the initial ramp for a correct definition of the parameters of fractional viscoelastic materials, Mechanics of Materials, vol.69, pp. 63-70, 2014.

[25] Podlubny I., Fractional Differential Equations, Academic Press, San Diego, 1999.

[26] Oldham K. B., Spainer J., The Fractional Calculus: Theory and applications of differentiation and integration to arbitrary order, Academic Press, New York, 1974.

[27] Caputo M., Linear models of dissipation whose $Q$ is almost frequency independent-II, Geophysical J. Royal Astronomic Society, Vol. 13, pp. 529-539, 1967. (Reprinted recently in: Fract. Calc. Appl. Anal., Vol. 11, No 1, pp. 3-14, 2008).

[28] Samko G. S., Kilbas A. A., Marichev O. I., Fractional Integrals and Derivatives: Theory and Applications, New York (NY): Gordon and Breach: 1993.

[29] Scott Blair G. W., Caffyn J. E., An application of the theory of quasiproperties to the treatment of anomalous strain-stress relations, The Philosophical Magazine, Volume 40, n. 300, pp. 80-94, January 1949.

[30] Mainardi F., Fractional Calculus and Waves in Linear Viscoelasticity, Imperial College Press-World Scientific Publishing Co. Pte. Ltd., London, 2010.

[31] Di Paola M., Pinnola F. P., Zingales M., Fractional multi-pahse hereditary materials: Mellin transform and multi-scale fractances, Proceedings of ECCOMAS 2012 - European Congress on Computational Methods in Applied Sciences and Engineering, e-Book Full Papers, pp. 4735-4745.

[32] Di Paola M., Pinnola F. P., Zingales M., Fractional differential equations and related exact mechanical models, Computers and Mathematics with Applications, Volume 66 (5), pp. 608-620, 2013.

[33] Curie M. J., Recherches sur la conductibilit des corps cristallises, Annales de chimie et de physique, ser. 6, Volume 18, pp. 203-269, 1889.

[34] Outstaloup A., La drivation non entire: thorie, synthse et applications, Paris: Herms, 1995.

[35] Jesus I. S., Tenreiro Machado J.A., Development of fractional order capacitors based on electrolyte processes, Nonlinear Dynamics, vol. 56, pp.4555, 2009 